\title{
Cognitive science - future challenges of an interdisciplinary field
}

\section{Eddy J. Davelaar*}

Birkbeck College, Universitiy of London, London, UK *Correspondence: e.davelaar@bbk.ac.uk

Cognitive Science has a long tradition of being interdisciplinary, not so much driven by the otherwise noble spirit of facilitating cross-talk, but by the very necessity to integrate multiple perspectives when tackling one of life's most complex questions: what is the mind and how does it work? As a global endeavour, Cognitive Science was shaped by many other disciplines, such as philosophy, biology, mathematics, physics, psychology, anthropology, linguistics, and sociology. The history of Cognitive Science is replete with examples of how collaborations across the disciplinary divide have pushed the field forward to unforeseen heights and of how new subfields emerged from such interactions.

Cognitive Science is a discipline with numerous successes, from understanding the limits of the human mind to its adaptive abilities in novel environments. The technological advancements in computing and the development of sophisticated laboratory software and hardware empower the scientist with tools to further unlock hidden wonders. Recent progress and debate have revived interest in the existence of an unconscious mind, a mind that seems to exhibit better information processing capability than the conscious mind.

Apart from moving swiftly forward, it is good practice to pause for breath and reflect. I once read in an announcement for an event that the aim of Cognitive Science is to reverse-engineer human intelligence. This announcement struck me as odd given that empirical scientists aim to understand the workings of the mind by subjecting it to various manipulations in controlled environments or by observing its behaviour in various uncontrolled environments. The resulting theoretical understandings mostly differ in the implementational details obtained from actual reverse engineering. Cognitive Science is first and foremost a science of the mind. It is true that the question of "what is a mind?" brings about heated debates that were already held at the very inception of the field. Does my cat have a mind? What about my occipital cortex or my genome? Does a society of individuals have a mind in the same way as does my brain with all its individual neurons?

Despite the many successes in understanding the workings of the mind, many influential theories do not take into account the influence of the environment in which the mind operates. The environment can be internal, such as bodily functions, or external such as nature, other individuals, or moving objects. Human beings are not the product of their environments, nor solely driven by an inherited script for life. The first great challenge for Cognitive Science is to include the interaction between the human being and its environment. For example, how is it possible that some highly intelligent people fail on tasks performed under exam situations?

Theories of human cognition have typically been applied to isolated (laboratory) situations, in which statistical techniques are used to address central tendencies. In this light, the second challenge is to aim to go beyond means and understand not only how variability in performance differs within and across individuals, but also across the lifespan. This will most likely involve employing statistical methods that are used in related disciplines or even developing new methods. Creating technical advances is not new to us, but the mindset of describing the generic individual under different circumstances is a particular strong bias, which we need to overcome in order to understand the unique individual as well as the similarities across individuals.

From the very beginning, questions in Cognitive Science were tackled through the development of models of the human mind. These models could be framed as a box-and-arrow diagram indicating how information flows through the system, of which the structure was deduced from psychological or biological experiments. Depending on the schooling of the scientist, the models could even be cast as a set of mathematical equations that focus on the assumed relevant information that underlies the behaviour of the mind. With the increased complexity of the equations and the exponential growth of interactions among the equations, certain models are only able to show predictions by computer simulation. The technological development in cognitive modelling in recent years has produced many new techniques that allow the scientist to tackle complex questions of model comparison.

Although cognitive modelling is an important research methodology, in order to understand the phenomenon that a computer program simulates, it is necessary to compare and contrast different implementations. The strength of Cognitive Science is in the application of different types of models to understand the complexity of the same phenomenon. The future of mathematical modelling within Cognitive Science lies not only in the endeavour of interfacing between levels of description, but also in the use of comparative metamodelling. Just like a meta-analysis of empirical work summarises and quantifies the effects obtained in experiments, so will a meta-model quantify the core principles, or laws, that are needed to understand the phenomenon. Therefore the third challenge I see is to work more collaboratively within and across cognitive topics with the aim of moving toward an interdisciplinary science that is also integrative.

From a philosophical perspective, Cognitive Science is filled with interesting questions. If thinking is the action in which the mind computes and computation involves the manipulation of symbols or representations, then the question is whether Cognitive Science is focusing on uncovering the computations of the mind or in mechanising the mind itself? If thinking is more than computation, is it possible that knowledge can be acquired without the involvement of computation? If 
Cognitive Science is truly involved in reverse engineering the mind, what does it mean to say that the mind is an emergent property? In empirical work, we try to infer the computations by means of manipulating a wide range of variables. In computational work, we go further and investigate how several uncovered computations interact given the various manipulations. What are the consequences of different philosophies about the mind on the methodologies employed, and does employing a certain methodology imply a particular philosophical conviction? As Cognitive Science is chronologically older than the new generation of scientists, an important challenge in the years to come is to revisit and understand the intricacies of the foundations of this multidisciplinary field. Great institutions tend to forget where they came from while moving swiftly forward, only to find themselves reaching a place which the founders would call the beginning. In this world of informationoverload, it is too easy to not see the trajectory and fall prey to confusing fashion with progress. Therefore, in my opinion, the greatest challenge for Cognitive Science is to remind ourselves about our aims and objectives and thereby keep the science of the mind moving forward and breaking new frontiers.

Received: 23 February 2010; accepted: 05 March 2010; published online: 29 March 2010.

Citation: Davelaar EJ (2010) Cognitive science - future challenges of an interdisciplinary field. Front. Psychology 1:7. doi: 10.3389/fpsyg.2010.00007

This article was submitted to Frontiers in Cognitive Science, a specialty of Frontiers in Psychology.

Copyright $\odot 2010$ Davelaar. This is an open-access article subject to an exclusive license agreement between the authors and the Frontiers Research Foundation, which permits unrestricted use, distribution, and reproduction in any medium, provided the original authors and source are credited. 